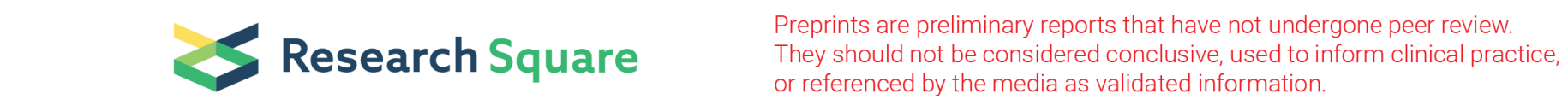

\title{
Evidence of Neuroplasticity: A Robotic Hand Exoskeleton Study for Post-Stroke Rehabilitation
}

\author{
Neha Singh \\ Indian Institute of Technology Delhi \\ Megha Saini \\ Indian Institute of Technology Delhi \\ Nand Kumar \\ All India Institute of Medical Sciences \\ M.V. Padma Srivastava \\ All India Institute of Medical Sciences \\ Amit Mehndiratta ( $\square$ amit.mehndiratta@keble.oxon.org ) \\ Indian Institute of Technology Delhi https://orcid.org/0000-0001-6477-2462
}

\section{Research}

Keywords: Stroke, Neurological rehabilitation, Wrist, Metacarpophalangeal joint, Robotic exoskeleton, Transcranial Magnetic Stimulation, corticalexcitability

Posted Date: September 3rd, 2020

DOI: https://doi.org/10.21203/rs.3.rs-67841/v1

License: (c) (i) This work is licensed under a Creative Commons Attribution 4.0 International License. Read Full License

Version of Record: A version of this preprint was published at Journal of NeuroEngineering and Rehabilitation on May 6th, 2021. See the published version at https://doi.org/10.1186/s12984-021-00867-7. 


\section{Abstract}

Background: A novel electromechanical robotic-exoskeleton was designed in-house for rehabilitation of wrist joint and Metacarpophalangeal (MCP) joint.

Objective: The objective was to compare the rehabilitation effectiveness (clinical-scales and neurophysiological-measures) of robotic-therapy training-sessions with dose-matched control in patients with stroke.

Methods: An observational pilot study was designed with patients within 2 years of chronicity. Patients received an intervention of 20 sessions of 45-minutes each, five days a week for four-weeks) in Robotic-therapy Group (RG) $(n=12)$ and conventional upper-limb rehabilitation in ControlGroup (CG) (n=11). Clinical-scales- Modified Ashworth Scale, Active Range of Motion, Barthel-Index, Brunstrom-stage and Fugl-Meyer scale (Shoulder/Elbow and Wrist/Hand component), and neurophysiological-measures of cortical-excitability (using Transcranial Magnetic Stimulation) -Motor Evoked Potential and Resting Motor-threshold, were acquired pre and post-therapy.

Results: RG and CG showed significant improvement in all clinical motor-outcomes $(p<0.05)$ except Modified Ashworth Scale in CG. RG showed significantly higher improvement over CG in Modified Ashworth Scale, Active Range of Motion and Fugl-Meyer (FM) scale and FM Wrist-/Hand component) ( $\mathrm{p}$ 0.05). Increase in cortical-excitability in ipsilesional-hemisphere was found to be statistically significant in RG over CG, as indexed by decrease in Resting Motor-Threshold and increase in amplitude of Motor Evoked Potential $(p<0.05)$. No significant changes were shown by the contralesional-hemisphere. Interhemispheric RMT-asymmetry evidenced significant changes in RG over CG ( $<<0.05$ ) indicating increased cortical-excitability in ipsilesional-hemisphere along with interhemispheric changes.

Conclusion: Neurophysiological-changes in RG could be most likely a consequence of plastic-reorganization and use-dependent plasticity. Robotic-exoskeleton training could significantly improve motor-outcomes and cortical-excitability in patients with stroke.

Registry number: IEC/NP-99/13.03.2015

\section{Introduction}

Stroke is one of the leading causes of mortality and morbidity worldwide (1). The ability to actively initiate extension movements at wrist and fingers against flexor-hypertonia is one of the key indicators of motor recovery (2),(3). Regaining hand-function and Activities of daily-living (ADL) is particularly impervious to therapy or rehabilitation pertaining to the complexity of motor-control needed for distal-joints (4). Conventional rehabilitation-therapy is time taking, labour-intensive and subjective, which with high clinical-load and absence of skilled resources gets difficult for the present medical and healthcare-system to provide appropriate or effective rehabilitation services (5).

Although rehabilitation with neuro-rehabilitation robots has shown encouraging clinical-results $(5,6,15,7-14)$, it is currently limited to a very few hospitals and not widely used because of associated high-cost and an infrastructural-requirement to station, size, complexity, set-up time, safety and usability restricting its success (16),(17),(18). Rehabilitation-strategies need to take into account the multifaceted nature of disability, which itself changes with time elapsed post-stroke and address with a multimodal-approach. Hence, the device needs to be flexible enough to accommodate a large patient-population. An effective rehabilitation device for hand should be able to facilitate a specific pattern of movements mirroring complex inter-joint coordination of hand with a patient-specific impairment, currently not integrated by the available devices.

In our previous work, we designed a robotic-hand exoskeleton for rehabilitation of the wrist and MCP (Metcarpo-phallengeal) joint, to synchronize wrist-extension with finger-flexion and wrist-flexion with finger-extension, mimicking ADL (19). With simple and easy-to-operate exoskeleton for low-resource settings, the exoskeleton targets spasticity through a synergy-based rehabilitation approach while also maintaining patient-initiated therapy through residual muscle-activity for maximizing voluntary effort. The lightweight and portable device has shown evidence of improvement in quantitative motor clinical-outcomes in patients with chronic stroke (19).

The aim of the present study was twofold. The first objective was to assess the clinical effectiveness of the novel robotic-exoskeleton device (19) and the second is comparison of its clinical-effectiveness with conventional upper-limb rehabilitation. We hypothesized that the exoskeleton could show higher improvement of distal-function and cortical-excitability in patients with stroke as compared to conventional-rehabilitation.

\section{Materials And Methods}

More than 300 patients $(n>300)$ were screened in the out-patient clinic of the Department of Neurology, AllMS, New-Delhi over 3 years from July2016 to January-2019. Stroke diagnosis was established clinically in all patients. All clinical-assessments and standard-care were given to the patients with stroke by a trained physiotherapist.

\subsection{Patient Enrolment}


Twenty-seven patients $(n=27)$ were enrolled for the therapy and twenty-three patients $(n=23)$ completed the therapy successfully (all righthanded patients with stroke, age $=41.9 \pm 11.1$ years, Male:Female $=19: 4$ ), details in Figure-1 and Table-1. Patients were enrolled based on inclusion-criteria, age 18-70 years, having single unilateral chronic ischemic / hemorrhagic stroke within 3-24 months, Mini-Mental Scale of $\geq$ 23; Brunnstrom-stage (BS) > 4; Modified Ashworth Scale (MAS) 1, 1+, 2. All enrolled patients continued to have their routine medication and standard medical care during the therapy-sessions, as advised by the neurologist. Institutional Review Board (IRB) at All India Institute of Medical Sciences (AIIMS), New-Delhi, India, approved the study under protocol-number IEC/NP-99/13.03.2015. All the patients signed the written informed consent before enrolment.

Table 1

Details of patients with stroke enrolled in Robotic Group ( $n=12)$ and Control Group $(n=11)$

\begin{tabular}{|llllll|}
\hline Group & Gender (M/F) & $\begin{array}{l}\text { Stroke type } \\
\text { Ischemic/ Hemorrhagic }\end{array}$ & Ipsilesional site, Left/Right & $\begin{array}{l}\text { Age } \\
\text { (years) }\end{array}$ & Chronicity (months) \\
\hline Robotic-therapy & $10 / 2$ & $9 / 3$ & $5 / 7$ & $41.1 \pm 12.8$ & $13.8 \pm 9.1$ \\
Control & $9 / 2$ & $8 / 3$ & $5 / 6$ & $42.7 \pm 9.3$ & $10.3 \pm 5.0$ \\
\hline
\end{tabular}

\subsection{Study-Design}

The study was designed as an observational pilot study which included pre and post clinical-outcome measures of therapy-intervention designed for post-stroke rehabilitation. Once enrolled, patients were then manually-randomized under two groups- Robotic-therapy group (RG) and ControlGroup (CG). Patients in RG underwent total 20 therapy-sessions, 5 days a week for 4-weeks, along with conventional physiotherapy-training. Patients in CG underwent conventional physiotherapy-sessions determined based on individual clinical-requirements. The same therapist provided therapy-sessions to all patients in both groups.

\subsection{Data Collection}

\subsubsection{Clinical scale measures}

All the participants underwent clinical-assessment (pre and post-therapy) for measuring the level of spasticity by Modified Ashworth Scale (MAS 0-4), range of voluntary wrist-movement defined in terms of Active Range of motion of wrist (AROM 00-700) as measured by a goniometer, stage of stroke recovery Brunnstrom-Stages (1-7), ADL measured by Barthel-Index (0-100) and functional and sensorimotor-control of upperlimb as measured by Fugl-Meyer Scale (0-66).

\subsubsection{Cortical-excitability measures using TMS}

Patients were allowed to sit comfortability in the chair, kept forearm pronated, elbow-joint at $90-120^{\circ}$ flexion, wrist-joint at a neutral position and fingers at rest. Single-pulse Transcranial Magnetic Stimulation (TMS) was given to evoke the Motor Evoked Potential (MEP) signal, using a flat 70 mm figure-of-eight coil (type D70 (AC), serial no. 0326, Magstim Rapid2, Magstim, UK), at the cortical-representation of the Extensor Digitorum Communis (EDC) muscle (on contralateral motor-cortex with reference to the EEG cap) of the ipsilesional and contralesional-hemisphere. Cortical-excitability was measured in terms of Resting motor-threshold (RMT) and Motor Evoked Potential (MEP)-amplitude using TMS over ipsilesional and contralesional-hemisphere according to the standard-protocol (20). Resting Motor-threshold was defined as the minimum intensity of TMS required to elicit a MEP in target contralateral-muscle in 5/10 trials, recorded in EMG, over the muscle cortical-representation in the primary motor-cortex. MEP encapsulates information relevant to the cortical-excitability of the brain, conduction, and functional-integrity of the corticospinal-tract (21). MEP should be $\geq 50 \mu v$ peak-to-peak amplitude at the hotspot in 5/10 consecutive trials.

\subsection{Robotic therapy-sessions}

An electromechanical robotic-exoskeleton was developed for rehabilitation of wrist-joint and fingers-joint (19) (Figure-2). It synchronizes wristextension with finger-flexion and wrist-flexion with finger-extension, a common pattern encountered in ADL. Device is actively-initiated by Electromyogram (EMG) and provides interactive adaptive performance-biofeedback in real-time. Device was safe, user-friendly and patientcentric with customizable motion-parameters (as per the clinical-presentation: i) initial-position for range of motion (ROM), ii) final-position for ROM, iii) speed, iv) residual muscle-activity and v) height of finger-support. All sessions were given at the hospital set-up under the supervision of an expert clinician. Therapy-protocol consisted of total 20 sessions, each of 45-min over 4-weeks. Each 45-minute robotic-therapy session consisted of approximately 250 -trials of 10 -seconds each. Patients were advised to take a 5 -min break for rest in between the therapy if there is a feeling of pain or fatigue. For details on exoskeleton design and protocol, please refer (19).

\subsection{Data analysis}

Data analysis was done in MATLAB R2018a (MATHWORKS). The data were tested for normality using Shapiro-Wilk test and was found that clinical-measures were not normally distributed in CG. Hence, non-parametric Wilcoxon signed-rank were used for intragroup-comparison of 
differences in post-pre-therapy within the group and non-parametric Man-Whitney tests were used for intergroup-comparison of RG and CG. Interhemispheric-asymmetry for pre and post-therapy measures were calculated and was tested using Wilcoxon signed-rank test. Two-way repeated measure ANOVA was applied to assess the effect of time (two levels-pre and post) and side (two levels-ipsilesional and contralesional) on RMT. Regression and correlation-analyses were performed to investigate the relationship of recovery parameters TMS neurophysiologicalparameter with clinical-outcome. A p-value $<0.05$ was considered as significant. MAS score of $1,1+, 2,3,4$ was mapped as $1,1.5,2,3,4$ for all statistical calculation purpose, respectively as by Rong et. al(13).

\section{Results}

All patients well tolerated the robotic therapy-sessions and completed in 30-34 days. There were no differences in the pre-therapy measures in terms of clinical-scales and MEP signal among both the intervention-groups $(p>0.05)$. One patient $(n=1)$ in $R G$ and three patients ( $n=3)$ in CG could not complete the therapy, thus data were excluded from further analysis. At pre-therapy measurements, MEP was evoked only for 9 patients $(R G=4, C G=5)$ out of total 23 in ipsilesional-hemisphere, and for all patients in contralesional-hemisphere.

\subsection{Comparison of Clinical-scales}

Post-therapy, all clinical-scales in both groups did show significant changes in improvement, except for MAS in CG. However, all clinical-scales (MAS, AROM, FM, and FMW/H) in RG showed statistically significant changes over CG. MAS in RG changed from $1.75 \pm 0.26$ to $1.29 \pm 0.33$ and in CG from $1.86 \pm 0.5$ to $1.59 \pm 0.66$ showing a significant decrease in spasticity at wrist-joint in RG and not in CG (RG-p=0.0009, CG-p=0.12) with significant intergroup changes ( $\mathrm{p}=0.03$ ) (Table-2). ADL, as indexed by AROM and $\mathrm{BI}$, in both groups showed statistically significant differences. AROM significantly improved in both the groups, from $150 \pm 9.70$ to $34.580 \pm 14.50$ in RG ( $p=0.0004)$ and from $13.60 \pm 7.70$ to $200 \pm 8.060$ in CG $(p=0.002)$. However, RG manifested statistically significant sensorimotor-scores as compared to CG as intergroup-comparison did evidence significant differences $(p=0.02)$ (Table-2). BI changed from $74.17 \pm 12.4$ to 89.17 \pm 7.93 in $R G(p=0.0009)$ and from $69.54 \pm 12.9$ to $82.72 \pm 14.3$ in $C G$ $(p=0.0009)$; the intergroup-comparison did not show any significant differences $(p=0.82)$. BS showed statistically significant differences in both groups, RG changed from $3.67 \pm 0.78$ to $4.83 \pm 0.94$ ( $p=0.0004$ ) and CG changed from $3.72 \pm 1$ to $4.45 \pm 1.2$ ( $p=0.015$ ). The intergroup-comparison did not show significant differences between both groups $(p=0.311)($ Table-2).

FMU/L scores measure sensorimotor-control gain in both groups after the intervention. FMU/L for RG changed from $36 \pm 7.78$ to $50.25 \pm 6.59$ $(p=0.0004)$ and from $37.45 \pm 9.1$ to $45.45 \pm 9.7$ for CG ( $p=0.0009)$. RG manifested statistically significant improvement in sensorimotor-scores as compared to CG with significant differences in intergroup-comparison ( $p=0.039)$ (Table-2). For the proximal-part-Shoulder/Elbow component of FMS/E, both groups showed statistically significant increase, RG changing from $26.2 \pm 5.6$ to $33.5 \pm 3.8$ ( $p=0.0009)$ and from $26 \pm 7.07$ to $29.8 \pm 7.08$ in $C G(p=0.002)$. However, the intergroup-comparison did not show any significant differences $(p=0.13)$. For the distal-part Wrist/Hand component of FM (FMW/H), both groups showed statistically significant increase, in RG changing from $9.7 \pm 2.7$ to $16.6 \pm 4.3$ ( $p=0.0004)$ and in CG changing from $11.45 \pm 2.9$ to $15.18 \pm 3.6$ ( $p=0.0009)$. RG manifested statistically significant sensorimotor-improvement in intergroupcomparison over CG ( $\mathrm{p}=0.012)$ (Table-2). 
Table 2

Comparison of clinical-scales and cortical-excitability (in ipsilesional and contralateral-hemisphere) in Robotic Group with Control Group

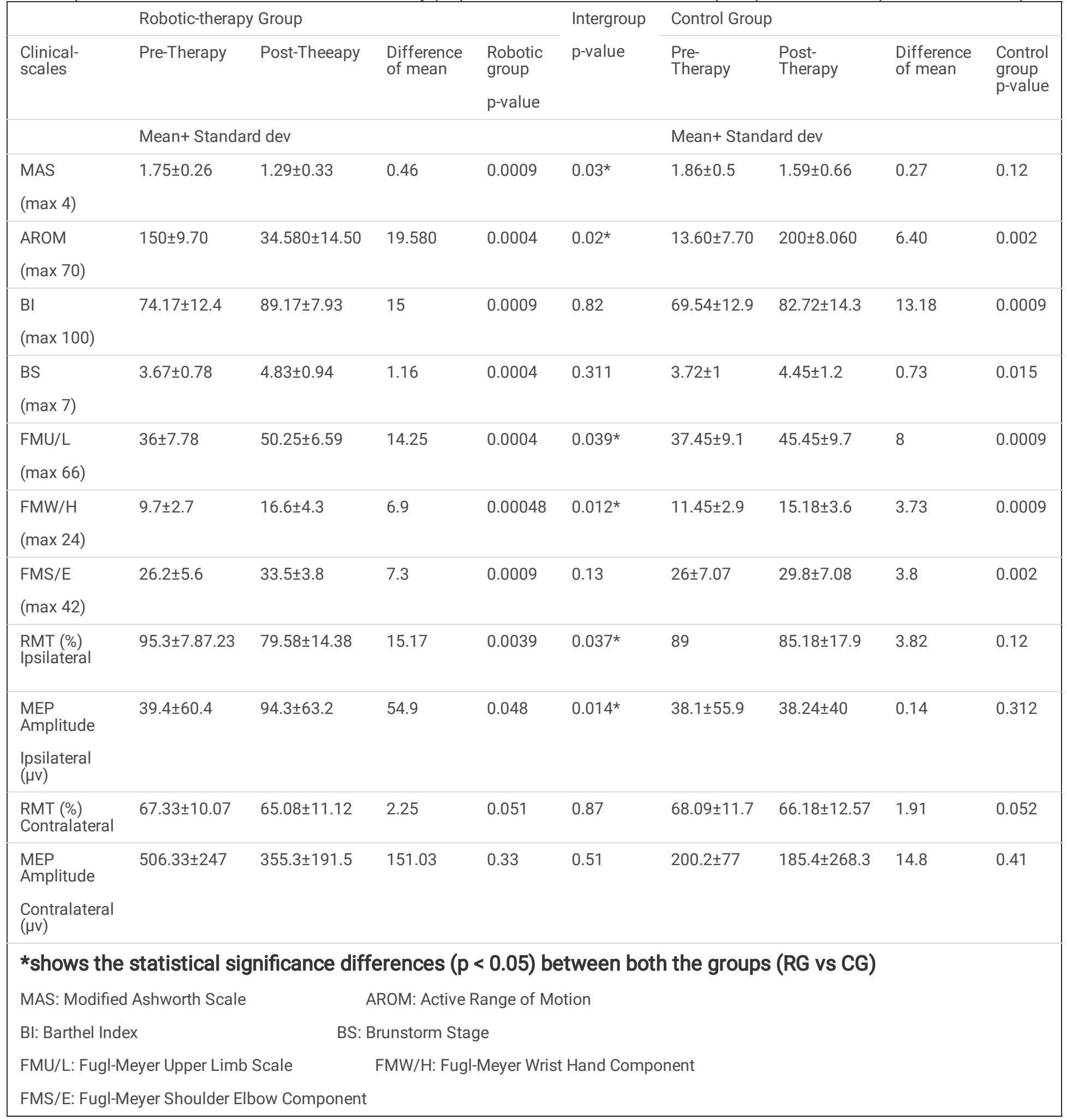

\subsection{Comparison of Cortical-excitability}

\subsubsection{Ipsilesional-hemisphere}


MEP, in some patients with stroke, is not recordable even after delivering TMS-stimuli at the highest possible intensity due to lower corticalexcitability. In those patients with no MEP recorded, RMT is taken as value of 100, as has been suggested in literature. Change in RMT showed statistically significant differences in post-therapy in RG as compared to CG. Post-therapy, in RG showed a significant decrease in RMT from $95.3 \pm 7.87 .23$ to $79.58 \pm 14.38$ ( $p=0.0039$ ) (indicating increase in cortical-excitability), whereas CG showed a decrease from 89 to $85.18 \pm 17.9$ $(p=0.12)$ (Table-2). RG also evidenced a significant increase in MEP-amplitude (indicating increase in cortical-excitability) from $39.4 \pm 60.4 \mu \mathrm{v}$ to 94.3 $\pm 63.2 \mu \mathrm{v}(\mathrm{p}=0.048)$. In CG, MEP almost remained same $(\sim 38 \mu \mathrm{v})$ pre-to-post-therapy $(\mathrm{p}=0.312)$. RG thus demonstrated a significant increase in cortical-excitability in intergroup-comparison over CG, as evidenced by decrease in RMT ( $p=0.037)$ and increase in MEP-amplitude ( $p=0.0142$ ) (Table-2).

In this study, $~ 54 \%$ of patients in CG did not evoke MEP. Approximately $~ 67 \%$ of patients in RG too did not evoke MEP at the pre-therapy measurements, most of which later evoked post-therapy showing the therapeutic-effectiveness of the exoskeleton. It is worth-noting that in RG, measurable MEP was evoked only in 4/12 patients at the pre-therapy measurements, however, post-therapy MEP was observed in $9 / 12$ patients, indicating a considerable increase in cortical-excitability in 5 patients. In CG, MEP was evoked only in 5/11 patients at the pre-therapy measurements and was observed in 6/11 patients post-therapy.

\subsubsection{Contralesional-hemisphere}

There were no significant changes shown by the contralesional-hemisphere. Both RG and CG evidenced minimal differences in RMT (mean increase of $\sim 2 \%$ in both groups) (Table-2). RG showed decrease from 67.33 \pm 10.07 to $65.08 \pm 11.12$ ( $p=0.051$ ) and CG from $68.09 \pm 11.7$ to $66.18 \pm 12.57$ ( $p=0.052)$. Intergroup-comparison too did not show statistically significant differences ( $p=0.87)(T a b l e-2)$. MEP-amplitude in RG decreased from $506.33 \pm 247$ to $355.3 \pm 191.5 \mu \mathrm{v}(\mathrm{p}=0.33)$ and from $200.2 \pm 77 \mu \mathrm{v}$ to $185.4 \pm 268.3 \mu \mathrm{v}$ in CG ( $\mathrm{p}=0.41)$. MEP-amplitude observed a considerable decrease (mean $151 \mu \mathrm{v}$ ) in RG indicating changes in cortical-excitability, as compared to CG (mean 15 $\mu \mathrm{v}$ ). The inter-group comparison however, was not statistically significant between both the groups $(p=0.51)($ Table-2).

\subsubsection{Inter-hemispheric differences and asymmetries}

The effect of robotic-exoskeleton training on cortical-excitability was assessed within both hemispheres. RG showed statistically significant differences between ipsilesional and contralesional-sides as one factor and time points- pre and post-therapy as another factor on RMT $(p=0.049, F=4.08)$, evidencing the dependence of time and hemisphere sides on each other. However, CG did not show any statistical differences $(p=0.06, F=3.68)$.

RG also evidenced a statistically significant reduction in interhemispheric-RMT asymmetry as measured by the ratio of RMT for two hemispheres $\left(\mathrm{RMT}_{\text {asymm }}=\mathrm{RMT}\right.$ Ipsilesional / RMT contralesional) from pre-to-post-therapy. RG showed a decrease in RMT asymm from $1.43 \pm 0.21$ to $1.25 \pm 0.31$ (mean decrease of $0.18, p=0.012$ ), however, CG showed a decrease from $1.33 \pm 0.30$ to $1.30 \pm 0.28$ (mean decrease of 0.03 , $p=0.59$ ), indicating a trend of normalization of RMT-asymmetry (RMT asymm should decrease as ipsilsesional RMT should be decreased from pre-to-post) over the duration of intervention in RG. RG also manifested statistically significant changes in intergroup-comparison over CG ( $p=0.028)$. The relative change in interhemispheric-RMT asymmetry-ratio ( $\Delta$ RMTasymm-ratio) changed with RG having a mean increase of $0.12 \pm 0.14$ and $C G$ a mean increase of $0.011 \pm 0.1(p=0.028)$, indicating the extent of normalization of RMT-asymmetry over the duration of intervention in RG as compared to CG (Table-3). 
Table 3

Details of each patient's RMT interhemispheric-Asymmetry (RMTasymm), relative improvement in RMT ratio $\left(\Delta \mathrm{RMT}_{\text {asymm }}\right.$ ratio), decrease/improvement in ipsilesional RMT ( $\Delta$ RMTipsi), and relative improvement in Fugl-Meyer $($ Wrist and Hand) $(\Delta$ FMW/H) of both groups with the mean \pm standard deviation

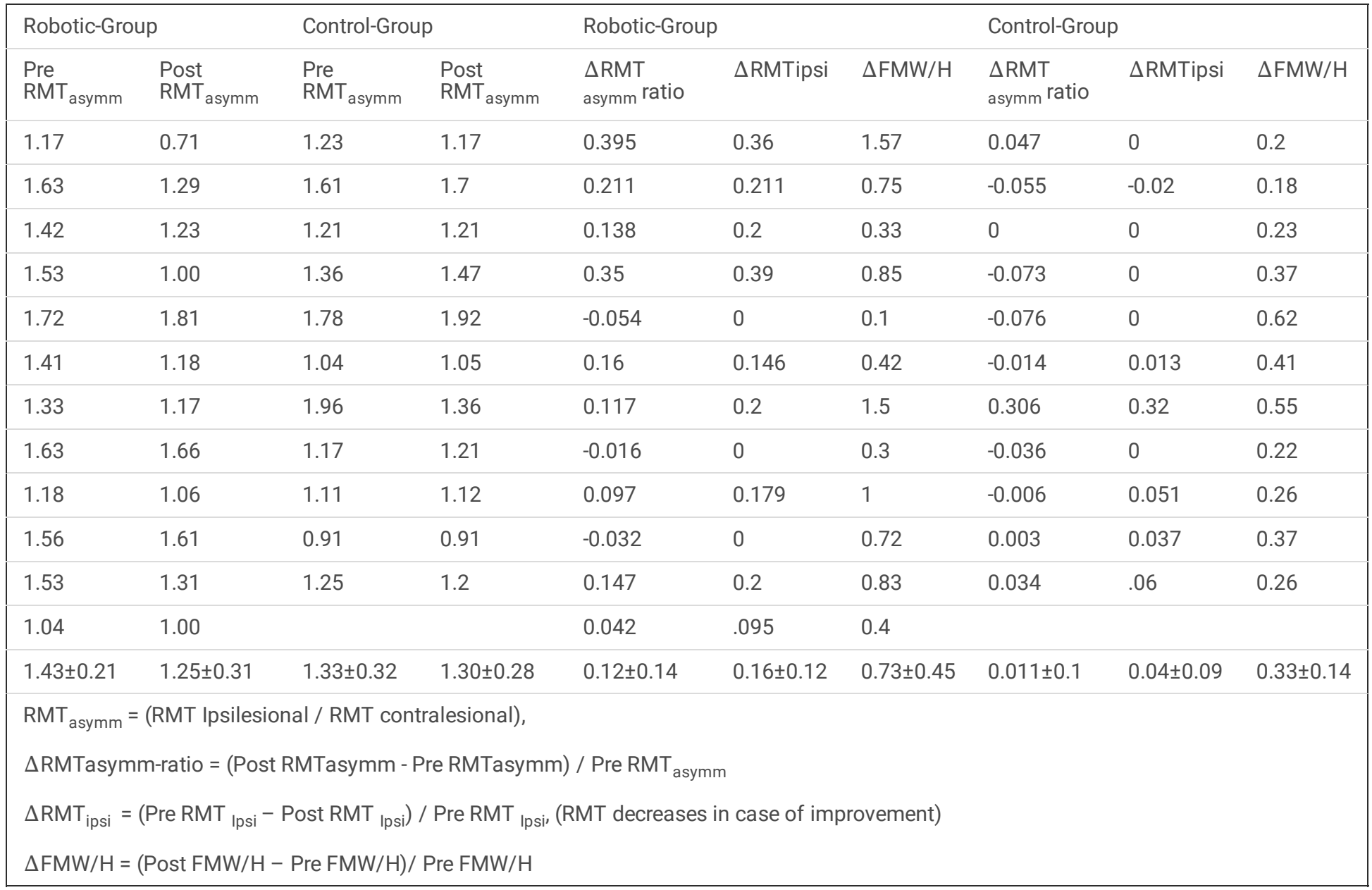

\subsection{Relationship between TMS neurophysiological-measures and clinical-outcome}

The recovery parameters from TMS-measures denoting the change from pre-to-post-therapy were observed to be strongly correlated with the relative change/improvement in distal motor-outcome $(\Delta \mathrm{FMW} / \mathrm{H})$. The first parameter, relative change in RMT in the ipsilesional-hemisphere ( $\Delta$ RMTipsi) was significantly different for both the groups $(p=0.0235)$ with a mean increase of $0.16 \pm 0.12$ in $R G$ and $0.04 \pm 0.09$ in $C G$. The linearregression analysis indicated that $\Delta \mathrm{RMT}_{\mathrm{ipsi}}$ (as predictive/independent-variable) could correlate with $\Delta \mathrm{FMW} / \mathrm{H}$ (as dependent-variable) and could predict $\Delta \mathrm{FMW} / \mathrm{H}$ in $\mathrm{RG}(\mathrm{r}=0.64, \mathrm{~F}=7.24, \mathrm{p}=0.022)$ (Table-3), indicating that increased cortical-excitability in the ipsilesional-hemisphere was correlated with the functional clinical-outcome. This correlation was not found in $\mathrm{CG}(\mathrm{r}=0.47, \mathrm{~F}=2.62, \mathrm{p}=0.13)$. The relationship between $\Delta \mathrm{RMT}$ ipsi and $\Delta \mathrm{FMW} / \mathrm{H}$ for both groups is shown in scatter-plot in figure-3a. The distal functional-outcome $\Delta \mathrm{FMW} / \mathrm{H}$ also showed significantly different results for both groups ( $p=0.012)$ with a mean increase of $0.73 \pm 0.45$ in $R G$ and $0.33 \pm 0.14$ in $C G$.

The second parameter, relative change in RMT-ratio ( $\triangle$ RMTasymm-ratio) was significantly different for both the groups ( $p=0.028)$. Similar to above, $\Delta$ RMTasymm-ratio (as predictive/independent-variable) was observed to be strongly correlated $\Delta \mathrm{FMW} / \mathrm{H}$ (as dependent-variable) ( $\mathrm{r}=0.6$, $\mathrm{F}=5.77, \mathrm{p}=0.03$ ) (Table-3), indicating that tendency towards extent of normalization RG could correlate and predict the clinical-outcome in RG. This correlation was not found in $\mathrm{CG}(\mathrm{r}=0.29, \mathrm{~F}=0.83, \mathrm{p}=0.38)$. The relationship between $\Delta \mathrm{RMTasymm}$-ratio and $\Delta \mathrm{FMW} / \mathrm{H}$ in both groups is shown in scatter-plot in figure-3b.

\section{Discussion}

The study demonstrated clinical and neurophysiological-changes in response to the robotic-exoskeleton (19) training compared to the conventional-rehabilitation. The clinical-scales showed improved changes in both RG and CG, however, increased cortical-excitability in the ipsilesional-hemisphere was shown only in RG. Five patients in RG, with the absence of MEPs at the pre-therapy measurements, showed the 
appearance of MEPs in the ipsilesional-hemisphere post-therapy. The improvement in RMT in ipsilesional-hemisphere showed a trend of normalization over the intervention and were also correlated with sensorimotor-functional improvement.

\subsection{Comparison of Clinical-scales of Robotic-therapy group with control-group}

The robotic-therapy was effective at releasing spasticity at the wrist-joint with $~ 26 \%(p=0.03)$ improvement over 14\% in CG. The regaining normal muscle-tone is considered as a predictor of recovery or the first-step in recovery (22) followed by an increase in muscle-strength and improvement in functional movements or ADL, finally leading to muscle-strength. In this study, AROM and Barthel-Index have been measured as the indicators of ADL (Table-2). Both groups showed significant improvement of AROM, however, RG showed significantly higher improvement of $130 \%$ over $47 \%$ in CG ( $p=0.02)$. AROM is one of an important parameters in evaluating ADL and increase in AROM of wrist could lead to greater participation in ADL (23). For Barthel-Index, both groups showed similar ( 20\%) improvement $(p=0.82)$. BI, non-specific crude discrete measure of $A D L$, measuring discretely for independence, partial-dependence and fully-dependence, and hence, even minimal improvement counts and increases the score by 5. A significant improvement in $\mathrm{BI}$ was observed for both the groups (RG \& CG). BI is not much reliable as scores might get affected depending on dominant/non-dominant side, thus, is not alone predictor for therapeutic-outcomes (24). Both groups showed significant improvement for BS, however, RG showed 32\% improvements over 20\% in CG (intergroup p $=0.31$ ) (Table-2). As BS measure the synergy pattern of joints, it depends mainly on various factors e.g. proximal and/or distal-joint condition of recovery, MAS and chronicity etc.

FMU/L, stroke-specific scale, is most reliable measure of sensorimotor-functionalities of the whole-arm (25). RG established significantly higher improvements of $\sim 40 \%$ over $~ 21 \%$ in CG $(p=0.039)$. In FMS/E, RG showed 28\% improvement over 15\% in CG. RG did not show significantly different results $(p=0.13)$ as expected as the intervention was not focused on proximal-component. However, for FMW/H distal-component, both groups showed significant improvement but RG showed significantly higher improvement of $~ 72 \%$ over $32 \%$ in CG ( $p=0.012$ ) because of intensive and repetitive-training of wrist and MCP. Similar improvement has also been reflected in proximal-joints in along with distal-joints robotic-training possibly because of compensatory muscle-activities from proximal-joints (12).

Thus, RG shows an overall increase in sensorimotor-ability and functionality evidenced by increase in FM, AROM and decrease in spasticity (MAS). Patients in RG showed more participation in ADL, reflected by an increase in $\mathrm{BI}$ and AROM of the wrist (23). Among the available wrist rehabilitative-devices and dose-matched conventional-intervention for rehabilitation of wrist in stroke, the reported gain in $\mathrm{FMW} / \mathrm{H}$ is $<4$ on a scale of 24 (12),(8),(26-28). In our study, the improvement in the FM score was $14.25(\mathrm{~W} / \mathrm{H} \sim 7)$. Any direct comparison with literature is, however, inappropriate considering different factors like chronicity of patients, lesion site, age of the patients, etc. in this study. HWARD(29) also showed improvement in $\mathrm{FMW} / \mathrm{H}$ ( 3.8), indicating the usefulness of synchronizing both wrist and MCP-joint movement in grasp and release.

\subsection{Comparison of Cortical-excitability of Robotic-therapy with the control-group}

Cortical-excitability in pre-therapy measurements was found to be lower in patients with stroke as observed by higher RMT and lower MEP (30), (31),(32),(33),(34). In some patients due to low cortical-excitability, MEP is not recordable even after delivering TMS-stimuli at the highest possible intensity (Maximum Stimulator Output at 100\%) (30),(31),(32),(33),(34). In those patients with no MEP recorded, RMT is taken as value of 100, as suggested in literature (35),(36). This study investigated specific impact of therapeutic-interventions on TMS neurophysiologicalparameters for cortical-pathways in the context of functional-gains of the hand motor-function.

\subsubsection{Ipsilesional and Contralesional-hemisphere changes}

At pre-therapy measures in the ipsilesional-hemisphere, only 39\% of the total patients' cohort in the study evoked MEP (Table-2). In RG, $67 \%$ of patients (8/12) did not evoke MEP. Out of these 8 (67\%) patients, $5(62 \%)$ patients later evoked MEP showing the therapeutic-effectiveness of the exoskeleton in RG. RG showed a significant decrease in RMT ( $p=0.0039)$ from pre-to-post-therapy-sessions which was not observed in CG ( $p=$ 0.12). RG showed 16\% improvement as compared to 4\% improvement in CG ( $p=0.037)$. Interestingly, RG showed significantly higher increase in MEP-amplitude ( $p=0.048$ ) post-therapy with increase of $\sim 140 \%$ (mean $=54.9 \mu \mathrm{v}$ ), whereas CG showed no such improvement. In the contralesional-hemisphere, MEP-amplitude showed a considerable decrease in both groups, though not significant, RG evidencing a considerable decrease of $\sim 30 \%$ (mean $=151.03 \mu \mathrm{v})$ and CG a decrease of only $\sim 7 \%$ (mean $=14.8 \mu \mathrm{v})$ with no significant differences $(p=0.51)($ Table-2).

Cortical-excitability measures are used as an objective investigative tool to measure the treatment responsiveness as it provide insights into membrane-excitability of neurons, conduction, and functional-integrity of corticospinal-tract and neuromuscular-junctions (37). The significant decrease in RMT and increase in MEP-amplitude in the ipsilesional-hemisphere demonstrates significant amount of increase in corticalexcitability (38), as was demonstrated in the RG versus CG. It can be interpreted that recovery of motor-function could most likely be a consequence of plastic-reorganization and use-dependent plasticity (38). Cortical-excitability and corticospinal-tract integrity have also been shown to be correlated with functional recovery potential in patients with chronic stroke (31) and exoskeleton-training appears to be beneficial in activating the ipsilesional-hemisphere for chronic patients (13.8 \pm 9.1 months). Activation of ipsilesional-hemisphere could indicate either vicariation of the loss of neural circuits or unmasking of pre-existing synapses or recruitment of perilesional areas in ipsilesional-hemisphere or exploitation of the preserved functional recovery reservoir in ipsilesional-hemisphere (35),(39),(40),(41). Further, a 30\% decrease in MEP- 
amplitude in contralesional-hemisphere over the duration of intervention might indicate a decrease in cortical-excitability, evidencing a trend towards restoring the Inter-Hemisphere Inhibition (IHI) balance in the motor-network between the two hemispheres(39),(40), however, it needs to be further evaluated in a larger cohort.

The cortical-excitability measures, usually, are acquired in pre and post-intervention mostly involving brain-stimulation studies. Examples are repetitive TMS, Transcranial Direct-Current Stimulation (tDCS)(42),(43), etc. or in a combination of brain-stimulation with other neurorehabilitation strategies like Constrain Induced Movement-Therapy (CIMT) (44) or mirror-therapy (45) or training(46),(47). However, studies evaluating the therapeutic-changes in the cortical-excitability using robotic-training intervention are very rare. To best of our knowledge, only two studies attempt to evaluate the effect of active robotic-training on changes in cortical-excitability, using commercially available devices, such as Lokomat-robot (lower-limb) (48) and ARMEO (upper-limb) (49).

\subsubsection{Specific five-patients in RG}

A very critical outcome of the therapy was that in RG, MEP was evoked in ipsilesional-hemisphere only for 4/12 patients at the pre-therapy measurements, however, MEP was later evoked for 9/12 patients after robotic-therapy. However, in CG, MEP was evoked only for $5 / 11$ patients and was later evoked for $6 / 11$ patients at post-therapy. Five specific patients in RG who did not evoke MEP at pre-therapy ( $0 \mu \mathrm{v})$ and later evoked MEP (mean $=136.6 \pm 38.48 \mu \mathrm{v})$, showed a decrease of RMT in ipsilesional-hemisphere (mean $=27 \pm 9.64)$, relative change RMT-ratio (mean $=$ $0.22 \pm 0.13$ ) and mean increase in clinical-scales (FMW/H: $7.8 \pm 2.38, \mathrm{BI}: 22 \pm 11.72, \mathrm{AROM}: 22 \pm 2.73$ ). These changes were relatively much higher than the changes in patients who had MEP evoked at pre-therapy measures. The appearance of MEP in five patients indicates that the robotic-therapy (of just four-weeks with 20 therapy-sessions) has an immense potential of training and reorganization of brain based on usedependent plasticity, for patients with chronic stroke. The increase in cortical-excitability and normalization of TMS neurophysiological-makers on the ipsilesional-side are also accompanied by greater recovery of hand-function, indexed by sensorimotor and functional recovery (by clinicalscales FMW/H, BI \& AROM). Indeed, the appearance of MEPs in ipsilesional-side could be a critical recovery marker in stroke recovery.

\subsubsection{Inter-hemispheric differences and asymmetries}

The diaschisis between ipsilesional-areas and intact neuronal-networks of contralesional-areas may disturb the cortical-excitability and connectivity-patterns of connected, remote, or primary-motor areas of contralesional-hemisphere (via transcallosal-fibers). The effect of roboticexoskeleton training on cortical-excitability of both hemisphere shows remodelling of the bilateral primary-motor areas in RG (time* sides $p=$ $0.049, F=4.08$ ) which is not shown in CG (time*sides $p=0.06, F=3.68$ ). The effect of exoskeleton-training shows the potential of the exoskeleton to accelerate the cortical-plasticity phenomena in favour of functional-restoration with changes in both ipsilesional and contralesional-hemispheres.

For cortical excitability to be increased in ipsilesional-hemisphere for patients with stroke, the ipsilesional-RMT should be decreased from pre-topost-therapy and hence, RMT asymm (RMT Ipsilesional/RMT contralesional) should decrease/approach normalization (35). Significant differences were observed between the groups when TMS-neurophysiological changes over the intervention was expressed in terms of the interhemisphericasymmetry ratio $\mathrm{RMT}_{\text {asymm }}$ indicating a significantly greater trend towards the normalization of asymmetry of TMS-measures in RG in response to exoskeleton-training than CG $(p=0.028)$. Also, the extent of normalization i.e. $\Delta$ RMTasymm-ratio showed a mean increase of $0.12 \pm 0.14$ in $R G$ and CG an increase of mean $0.011 \pm 0.1$ (Table-3). Normalization might indicate the recruitment of peri-lesional areas in the ipsilesionalhemisphere or exploitation of the preserved functional-recovery reservoir in the ipsilesional-hemisphere (35),(39),(40),(41). Normalization in response to therapy, in terms of TMS measures on the ipsilesional-side, has been shown to have a greater recovery of arm and hand function in acute, sub-acute and chronic stages (35).

\subsection{TMS neurophysiological improvement correlating the motor-outcome of both groups}

The amount of change in TMS neurophysiological-measures of corticomotor-pathways ( $\Delta \mathrm{RMT}_{\mathrm{ipsi}}$ and $\left.\Delta \mathrm{RMTasymm-ratio}\right)$ were found to be associated with the amount of improvement in functional motor-outcome during rehabilitation of the distal-part of upper-limb $(\Delta \mathrm{FMW} / \mathrm{H})$ (Figure-3). These parameters were significantly different for RG and CG $\left(\Delta \mathrm{RMT}_{\mathrm{ipsi}} \mathrm{p}=0.0235, \Delta \mathrm{RMTasymm}\right.$-ratio $\mathrm{p}=0.028$ and $\Delta \mathrm{FMW} / \mathrm{H} \mathrm{p}=$ 0.012). Greater improvement (decrease) in motor-threshold tend to show greater increases with clinical-outcome and was found to have strong positive statistical correlation with $\Delta \mathrm{FMW} / \mathrm{H}$ in $\mathrm{RG}\left(\Delta \mathrm{RMT}_{\text {ipsi }} \mathrm{r}=0.64, \mathrm{p}=0.022\right.$ and not in $\mathrm{CG} r=0.47, \mathrm{p}=0.13$ and $\Delta \mathrm{RMTasymm}$-ratio $\mathrm{r}=0.6, \mathrm{p}=$ 0.03 and not in CG $(r=0.29, p=0.38)$ (Figure-3). The improvement in RMT were most likely due to increased cortical-excitability of preserved motor-pathways with earlier studies in sub-acute and chronic stroke demonstrating correlation of improvement in TMS neurophysiologicalmeasures (improvement in RMT and normalization) with functional improvement (35),(50),(51). This also suggests the usefulness of TMSmeasures as an index of recovery in the ipsilesional-hemisphere (35). These neurophysiological-measures were obtained specifically from cortical-representation of EDC muscle, a clinically affected muscle, with a specific function which was involved in training with a roboticexoskeleton, whereas most clinical-measures do not necessarily require a particular muscle-group and measures motor-function in a broader sense.

Page 9/15 
Also, these neurophysiological-parameters individually establishes as a significant predictor $\left(\Delta R M T_{i p s i} r=0.64, F=7.24, p=0.022\right.$ and $\Delta$ RMTasymm-ratio $r=0.6, F=5.77, p=0.03)$ of functional rehabilitation-outcome of hand $(\Delta F M W / H)$ in $R G$, indicating that changes in corticalexcitability of ipsilesional-hemisphere could be used to predict the clinical-outcome, hence, emerging as critical recovery parameters to be considered and evaluated in larger data-samples. This might possibly be the plasticity markers predicting the responsiveness of chronic poststroke patients(49). Hence, the correlation and prediction of improvement in $\Delta \mathrm{FMW} / \mathrm{H}$ component by these muscle-specific neurophysiologicalmeasures comes as an evidence of task-specific rehabilitation of specific-muscle.

\subsection{Changes due to the device}

The exoskeleton training in RG induced an evident modulation in ipsilesional and contralesional-hemispheres. However, (significant) changes in CG were found to be limited only to the clinical-scales, and the changes in brain was specifically found only in the RG. An increase in corticalexcitability in the ipsilesional-hemisphere along with interhemispheric-normalization of $\mathrm{RMT}_{\text {asymm }}$ could point towards the recruitment of perilesional areas in ipsilesional-hemisphere or exploitation of the preserved functional recovery reservoir in the ipsilesional-hemisphere (35),(39), (40),(41). The decrease of RMT and change in RMT asymmetry from distal-muscle was also accompanied by functional markers-FMW/H evidencing sensorimotor-plasticity, functional recovery along with task-dependent rehabilitation. Greater magnitude of the neurophysiologicalchanges observed in RG, as compared to CG, may be attributed to the unique features of the device e.g. easy donning and doffing of the device for repositioning of hand, maximum finger-extension at baseline position to provide maximum stretch to reduce spasticity; and diverse attributes of device e.g bio-triggered, real-time adaptive performance-feedback, counteracting flexor-hypertonia against gravity, mimicking functional motion, user-friendly, simple design and patient-specific customizable features with different amount of sensory inputs (proprioceptive, visual, tactile) (19). In combination with the above features, the other unique feature of the device is that it allows the facilitation of a specific-pattern of movements mirroring complex inter-joint coordination of hand with a patient-specific impairment, currently not integrated in the available other devices with isolated-joint movements (52). The devices were able to simulate the movement pattern maintaining joint-coordination, especially at the distal-joints which could aid in translating the motor-improvements into ADL.

\section{Limitations}

Even though data are promising, the study had few limitations such as small sample-size and no long term follow-up of patients. As most of the patients at our quaternary hospital came from far places across India and it was not possible to follow-up with them once they have left NewDelhi.

\section{Conclusion}

The robotic-exoskeleton illustrated considerable improvement in motor clinical-outcomes and increased cortical-excitability in the ipsilesionalhemisphere in patients with stroke as compared to the control-group. This technology-based intervention has the potential to enhance the recovery of stroke neuro-rehabilitation.

\section{Declarations}

\section{Ethical Statement:}

Institutional Review Board (IRB) at All India Institute of Medical Sciences (AllMS), New-Delhi, India, approved the study under protocol-number IEC/NP-99/13.03.2015. All the patients signed the written informed consent before enrolment.

\section{Consent for Publication:}

All the patients signed the written informed consent for publication before enrolment.

\section{Availability of Data and Material:}

All data requests from the scientific community should be directed to the corresponding author for consideration.

\section{Competing Interest:}

The Author(s) declare(s) that there is no conflict of interest. 


\section{Funding:}

This work was financially supported by SERB, DST India (YSS/2015/000697).

\section{Authors Contributors:}

NS and AM conceptualized and designed the study. AM led the study and provided the scientific inputs. MS performed patient recruitment, physiotherapy and data collection. NK and PS provided the scientific inputs, clinical support and clinical resources for experiments. NS performed literature survey, coordinated experiments, data analysis, data interpretation, wrote the manuscript. AM reviewed the manuscript at multiple iteration with NS. All autors reviewed and approved the manuscript.

\section{Acknowledgment:}

The authors thank all patients who participated in the study.

\section{References}

1. Stroke Statistics | Internet Stroke Center [Internet]. [cited 2019 Aug 25]. Available from: http://www.strokecenter.org/patients/aboutstroke/stroke-statistics/

2. Kuo C-L, Hu G-C. Post-stroke Spasticity: A Review of Epidemiology, Pathophysiology, and Treatments. Int J Gerontol [Internet]. 2018 Dec 1 [cited 2019 Aug 23];12(4):280-4. Available from: https://www.sciencedirect.com/science/article/pii/S1873959818300073

3. Wolf SL, Winstein CJ, Miller JP, Taub E, Uswatte G, Morris D, et al. Effect of constraint-induced movement therapy on upper extremity function 3 to 9 months after stroke: The EXCITE randomized clinical trial. J Am Med Assoc [Internet]. 2006 Nov 1 [cited 2020 Jun 22];296(17):2095-104. Available from: https://pubmed.ncbi.nlm.nih.gov/17077374/

4. Yarossi M, Patel J, Qiu Q, Massood S, Fluet G, Merians A, et al. The Association Between Reorganization of Bilateral M1 Topography and Function in Response to Early Intensive Hand Focused Upper Limb Rehabilitation Following Stroke Is Dependent on Ipsilesional Corticospinal Tract Integrity. Front Neurol [Internet]. 2019 [cited 2019 Aug 22];10:258. Available from: http://www.ncbi.nlm.nih.gov/pubmed/30972004

5. Qian Q, Hu X, Lai Q, Ng SC, Zheng Y, Poon W. Early stroke rehabilitation of the upper limb assisted with an electromyography-driven neuromuscular electrical stimulation-robotic arm. Front Neurol. 2017;8(SEP):1-13.

6. Linder SM, Rosenfeldt AB, Reiss A, Buchanan S, Sahu K, Bay CR, et al. The home stroke rehabilitation and monitoring system trial: A randomized controlled trial. Int J Stroke. 2013;8(1):46-53.

7. Hu XL, Tong KY, Song R, Zheng XJ, Leung WWF. A comparison between electromyography-driven robot and passive motion device on wrist rehabilitation for chronic stroke. Neurorehabil Neural Repair. 2009;23(8):837-46.

8. Hu XL, Tong KY, Li R, Xue JJ, Ho SK, Chen P. The effects of electromechanical wrist robot assistive system with neuromuscular electrical stimulation for stroke rehabilitation. J Electromyogr Kinesiol [Internet]. 2012 Jun [cited 2018 Sep 6];22(3):431-9. Available from: http://www.ncbi.nlm.nih.gov/pubmed/22277205

9. Takahashi CD, Der-y ÃL, Le V, Motiwala RR, Cramer SC. Robot-based hand motor therapy after stroke. 2008;

10. Hu XL, Tong KY, Song R, Zheng XJ, Lui KH, Leung WWF, et al. Quantitative evaluation of motor functional recovery process in chronic stroke patients during robot-assisted wrist training. J Electromyogr Kinesiol [Internet]. 2009;19(4):639-50. Available from: http://dx.doi.org/10.1016/j.jelekin.2008.04.002

11. Song R, Tong KY, Hu X, Zhou W. Myoelectrically controlled wrist robot for stroke rehabilitation. J Neuroeng Rehabil. 2013;10(1):1-8.

12. Hu XL, Tong RKY, Ho NSK, Xue JJ, Rong W, Li LSW. Wrist Rehabilitation Assisted by an Electromyography-Driven Neuromuscular Electrical Stimulation Robot after Stroke. Neurorehabil Neural Repair. 2015;29(8):767-76.

13. Rong W, Li W, Pang M, Hu J, Wei X, Yang B, et al. A Neuromuscular Electrical Stimulation (NMES) and robot hybrid system for multi-joint coordinated upper limb rehabilitation after stroke. J Neuroeng Rehabil. 2017;14(1):1-13.

14. Nam C, Rong W, Li W, Xie Y, Hu X, Zheng Y. The effects of upper-limb training assisted with an electromyography-driven neuromuscular electrical stimulation robotic hand on chronic stroke. Front Neurol. 2017;8(DEC).

15. Hu XL, Tong KY, Wei XJ, Rong W, Susanto EA, Ho SK. The effects of post-stroke upper-limb training with an electromyography (EMG)-driven hand robot. J Electromyogr Kinesiol. 2013;23(5):1065-74.

16. Oujamaa L, Relave I, Froger J, Mottet D, Pelissier J-Y. Rehabilitation of arm function after stroke. Literature review. Ann Phys Rehabil Med [Internet]. 2009 Apr [cited 2017 Oct 8];52(3):269-93. Available from: http://www.ncbi.nlm.nih.gov/pubmed/19398398

Page $11 / 15$ 
17. Balasubramanian S, Klein J, Burdet E. Robot-assisted rehabilitation of hand function. Curr Opin Neurol [Internet]. 2010 Dec [cited 2019 May 22];23(6):661-70. Available from: https://insights.ovid.com/crossref?an=00019052-201012000-00019

18. Lee M, Rittenhouse M, Abdullah HA. Design Issues for Therapeutic Robot Systems: Results from a Survey of Physiotherapists. J Intell Robot Syst [Internet]. 2005 Mar [cited 2019 Aug 28];42(3):239-52. Available from: http://link.springer.com/10.1007/s10846-004-7194-y

19. Singh N, Saini M, Anand S, Kumar N, Srivastava MVP, Mehndiratta A. Robotic Exoskeleton for Wrist and Fingers Joint in Post-Stroke NeuroRehabilitation for Low-Resource Settings. IEEE Trans Neural Syst Rehabil Eng [Internet]. 2019 [cited 2019 Sep 27];1-1. Available from: https://ieeexplore.ieee.org/document/8846101/

20. Awiszus F. Chapter 2 TMS and threshold hunting. Suppl Clin Neurophysiol [Internet]. 2003 Jan 1 [cited 2019 Jul 10];56:13-23. Available from: https://www.sciencedirect.com/science/article/abs/pii/S1567424X09702053

21. Singh N, Saini M, Kumar N, Deepak KK, Anand S, Srivastava MVP, et al. Time-Frequency Analysis of Motor-Evoked Potential in Patients with Stroke vs Healthy Subjects: a Transcranial Magnetic Stimulation Study. SN Compr Clin Med. 2019 Oct;1(10):764-80.

22. Plantin J, Pennati G V., Roca P, Baron JC, Laurencikas E, Weber K, et al. Quantitative assessment of hand spasticity after stroke: Imaging correlates and impact on motor recovery. Front Neurol. 2019;10(JUL).

23. Schuster-Amft C, Van Kerckhoven L, Berse M, Verheyden G. Immediate effects of different upper limb robot-assisted training modes in patients after stroke: A case series. Schumacher U, editor. Cogent Med [Internet]. 2016 Sep 26 [cited 2019 May 22];3(1). Available from: https://www.cogentoa.com/article/10.1080/2331205X.2016.1240282

24. Barthel Index [Internet]. Available from: https://www.physio-pedia.com/Barthel_Index\#cite_note-2).

25. Lundquist CB, Maribo T. The Fugl-Meyer assessment of the upper extremity: reliability, responsiveness and validity of the Danish version. Disabil Rehabil [Internet]. 2017 Apr 24 [cited 2019 Sep 8];39(9):934-9. Available from: http://www.ncbi.nlm.nih.gov/pubmed/27062881

26. Shin J-H, Kim M-Y, Lee J-Y, Jeon Y-J, Kim S, Lee S, et al. Effects of virtual reality-based rehabilitation on distal upper extremity function and health-related quality of life: a single-blinded, randomized controlled trial. J Neuroeng Rehabil [Internet]. 2016;13(1):17. Available from: http://www.jneuroengrehab.com/content/13/1/17

27. Liao W, Wu C, Hsieh Y, Lin K, Chang W. Effects of robot-assisted upper limb rehabilitation on daily function and real-world arm activity in patients with chronic stroke: a randomized controlled trial. Clin Rehabil [Internet]. 2012 Feb 12 [cited 2018 Sep 6];26(2):111-20. Available from: http://www.ncbi.nlm.nih.gov/pubmed/21840917

28. Lee M-J, Lee J-H, Lee S-M. Effects of robot-assisted therapy on upper extremity function and activities of daily living in hemiplegic patients: A single-blinded, randomized, controlled trial. Technol Heal Care [Internet]. 2018 Aug 10 [cited 2018 Sep 6];1-8. Available from: http://www.ncbi.nlm.nih.gov/pubmed/30124459

29. Takahashi CD, Der-Yeghiaian L, Le V, Motiwala RR, Cramer SC. Robot-based hand motor therapy after stroke. Brain. 2008;131(2):425-37.

30. Chen R, Cros D, Curra A, Di Lazzaro V, Lefaucheur JP, Magistris MR, et al. The clinical diagnostic utility of transcranial magnetic stimulation: Report of an IFCN committee. Clin Neurophysiol. 2008;119(3):504-32.

31. Stinear CM, Barber PA, Smale PR, Coxon JP, Fleming MK, Byblow WD. Functional potential in chronic stroke patients depends on corticospinal tract integrity. Brain [Internet]. 2006 Nov 21 [cited 2019 May 12];130(1):170-80. Available from: http://www.ncbi.nlm.nih.gov/pubmed/17148468

32. Kim G-W, Won YH, Park S-H, Seo J-H, Ko M-H. Can Motor Evoked Potentials Be an Objective Parameter to Assess Extremity Function at the Acute or Subacute Stroke Stage? Ann Rehabil Med [Internet]. 2015 Apr [cited 2019 Aug 19];39(2):253. Available from: http://www.ncbi.nlm.nih.gov/pubmed/25932422

33. Amengual JL, Valero-Cabré A, de las Heras MV, Rojo N, Froudist-Walsh S, Ripollés P, et al. Prognostic value of cortically induced motor evoked activity by TMS in chronic stroke: Caveats from a revealing single clinical case. BMC Neurol [Internet]. 2012 Dec 8 [cited 2019 Aug 19];12(1):35. Available from: http://bmcneurol.biomedcentral.com/articles/10.1186/1471-2377-12-35

34. Takeuchi N, Tada T, Toshima M, Ikoma K. Correlation of motor function with transcallosal and intracortical inhibition after stroke. J Rehabil Med [Internet]. 2010 [cited 2019 Aug 20];42(10):962-6. Available from: https://medicaljournals.se/jrm/content/abstract/10.2340/165019770628

35. Koski L, Mernar TJ, Dobkin BH. Immediate and Long-Term Changes in Corticomotor Output in Response to Rehabilitation: Correlation with Functional Improvements in Chronic Stroke. Neurorehabil Neural Repair [Internet]. 2004 Dec 1 [cited 2019 Sep 1];18(4):230-49. Available from: http://journals.sagepub.com/doi/10.1177/1545968304269210

36. Huynh W, Vucic S, Krishnan A V., Lin CSY, Kiernan MC. Exploring the evolution of cortical excitability following acute stroke. Neurorehabil Neural Repair. 2016 Mar 1;30(3):244-57.

37. Escudero J V, Sancho J, Bautista D, Escudero M, López-Trigo J. Prognostic value of motor evoked potential obtained by transcranial magnetic brain stimulation in motor function recovery in patients with acute ischemic stroke. Stroke. 1998;29(9):1854-9.

38. Talelli P, Greenwood RJ, Rothwell JC. Arm function after stroke: Neurophysiological correlates and recovery mechanisms assessed by transcranial magnetic stimulation. Clin Neurophysiol [Internet]. 2006 Aug 1 [cited 2019 May 12];117(8):1641-59. Available from:

Page 12/15 
https://www.sciencedirect.com/science/article/pii/S1388245706000484?via\%3Dihub

39. Edwardson MA, Lucas TH, Carey JR, Fetz EE. New modalities of brain stimulation for stroke rehabilitation. Exp Brain Res. 2013;224(3):33558.

40. Dodd KC, Nair VA, Prabhakaran V. Role of the contralesional vs. Ipsilesional hemisphere in stroke recovery. Vol. 11, Frontiers in Human Neuroscience. Frontiers Media S. A; 2017. p. 469-469.

41. Du J, Yang F, Hu J, Hu J, Xu Q, Cong N, et al. Effects of high- and low-frequency repetitive transcranial magnetic stimulation on motor recovery in early stroke patients: Evidence from a randomized controlled trial with clinical, neurophysiological and functional imaging assessments. Neurolmage Clin [Internet]. 2019 [cited 2019 Sep 27];21:101620. Available from:

http://www.ncbi.nlm.nih.gov/pubmed/30527907

42. Kubis N. Non-Invasive Brain Stimulation to Enhance Post-Stroke Recovery. Front Neural Circuits [Internet]. 2016 [cited 2017 Dec 27];10:56. Available from: http://www.ncbi.nlm.nih.gov/pubmed/27512367

43. Solomons C, Shanmugasundaram V. A review of transcranial electrical stimulation methods in stroke rehabilitation. Neurol India [Internet]. 2019 [cited 2019 Aug 19];67(2):417. Available from: http://www.ncbi.nlm.nih.gov/pubmed/31085852

44. Henderson L, Paul M, David M, Deana G, Davalos B. THESIS THE USE OF REPETITIVE TRANSCRANIAL MAGNETIC STIMULATION AS AN ADJUNCT TO CONSTRAINT INDUCED THERAPY Submitted By [Internet]. 2013 [cited 2019 Aug 20]. Available from: https://mountainscholar.org/bitstream/handle/10217/81026/Henderson_colostate_0053N_12150.pdf?sequence=1

45. Kim J, Yim J. Effects of High-Frequency Repetitive Transcranial Magnetic Stimulation Combined with Task-Oriented Mirror Therapy Training on Hand Rehabilitation of Acute Stroke Patients. Med Sci Monit [Internet]. 2018 Feb 6 [cited 2019 Aug 20];24:743-50. Available from: http://www.ncbi.nlm.nih.gov/pubmed/29402879

46. Calabrò RS, Naro A, Leo A, Bramanti P. Usefulness of robotic gait training plus neuromodulation in chronic spinal cord injury: a case report. J Spinal Cord Med [Internet]. 2017 Jan 2 [cited 2019 Aug 20];40(1):118-21. Available from: http://www.ncbi.nlm.nih.gov/pubmed/27077568

47. Kang Y, Park H, Kim H, Lim T, Ku J, Cho S, et al. Upper extremity rehabilitation of stroke: Facilitation of corticospinal excitability using virtual mirror paradigm. J Neuroeng Rehabil [Internet]. 2012 Oct 4 [cited 2019 Aug 20];9(1):71. Available from:

http://www.ncbi.nlm.nih.gov/pubmed/23035951

48. Krishnan C, Ranganathan R, Kantak SS, Dhaher YY, Rymer WZ. Active robotic training improves locomotor function in a stroke survivor. J Neuroeng Rehabil [Internet]. 2012;9:57. Available from: http://www.pubmedcentral.nih.gov/articlerender.fcgi? artid=3480863\&tool=pmcentrez\&rendertype=abstract

49. Calabrò RS, Russo M, Naro A, Milardi D, Balletta T, Leo A, et al. Who May Benefit From Armeo Power Treatment? A Neurophysiological Approach to Predict Neurorehabilitation Outcomes. PM R. 2016 Oct 1;8(10):971-8.

50. Liepert J, Bauder H, Miltner WHR, Taub E, Weiller C. Treatment-Induced Cortical Reorganization After Stroke in Humans. Stroke [Internet]. 2000 Jun [cited 2019 Sep 1];31(6):1210-6. Available from: https://www.ahajournals.org/doi/10.1161/01.STR.31.6.1210

51. Traversa R, Cicinelli P, Pasqualetti P, Filippi M, Rossini PM. Follow-up of interhemispheric differences of motor evoked potentials from the "affected" and "unaffected" hemispheres in human stroke. Brain Res [Internet]. 1998 Aug 24 [cited 2020 Jul 20];803(1-2):1-8. Available from: https://pubmed.ncbi.nlm.nih.gov/9729235/

52. Lee SW, Landers KA, Park HS. Development of a biomimetic hand exotendon device (BiomHED) for restoration of functional hand movement post-stroke. IEEE Trans Neural Syst Rehabil Eng. 2014;22(4):886-98.

\section{Figures}




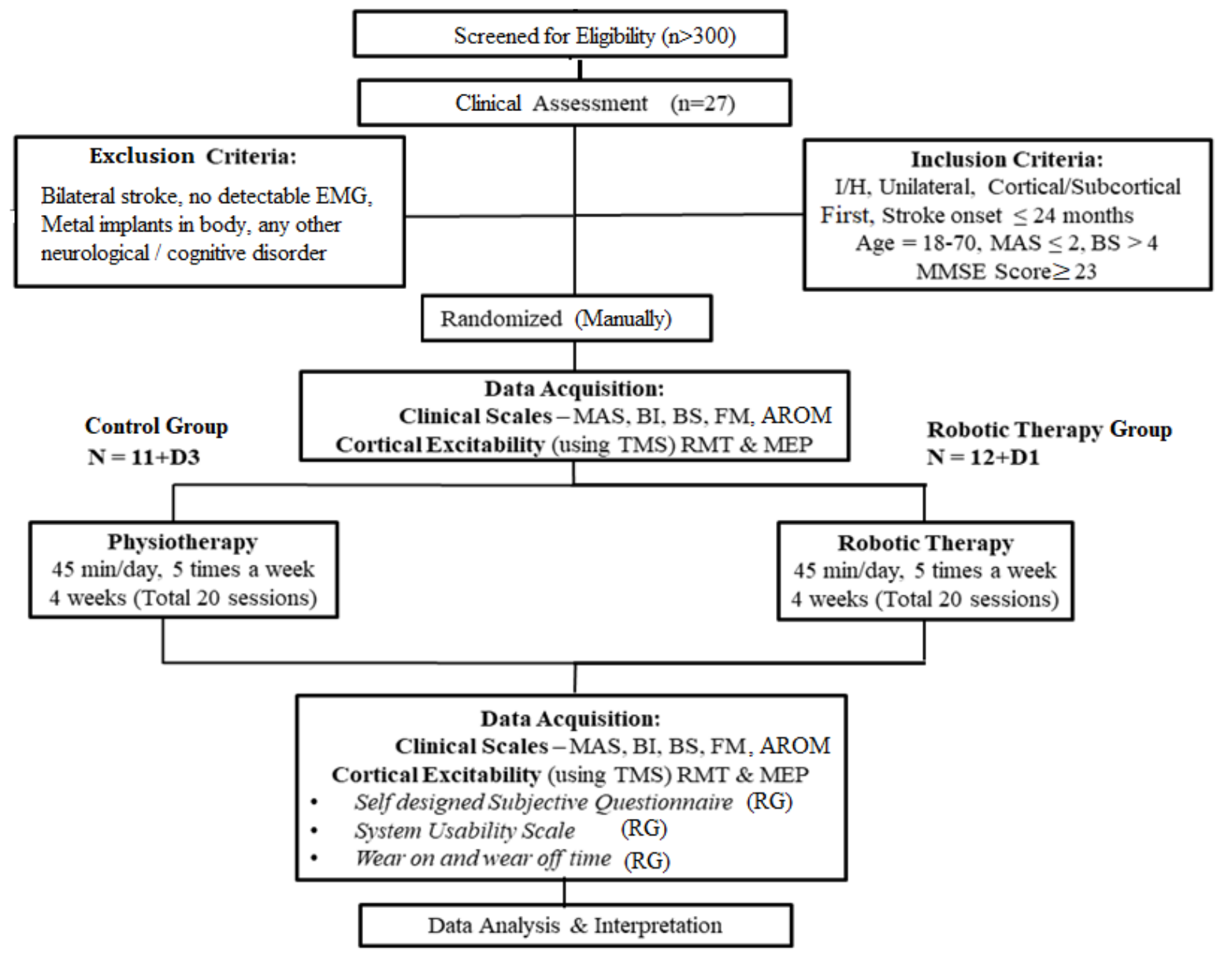

Figure 1

Patient Enrolment Consort, D indicates dropout

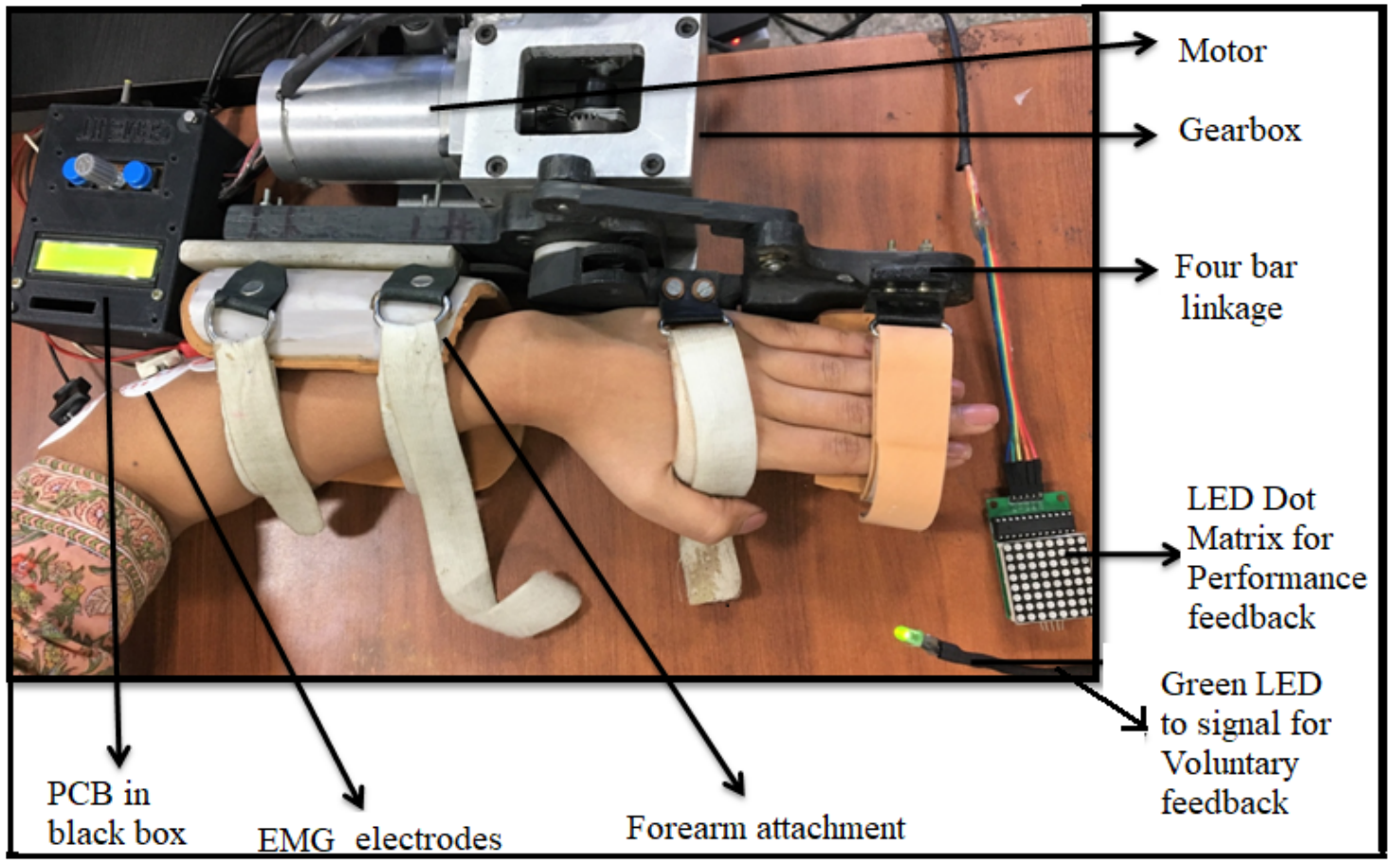

Figure 2

Whole set-up of exoskeleton with performance biofeedback, voluntary cue and PCB in the black control-box which also works as user-interface (refer Singh et al (19)) 


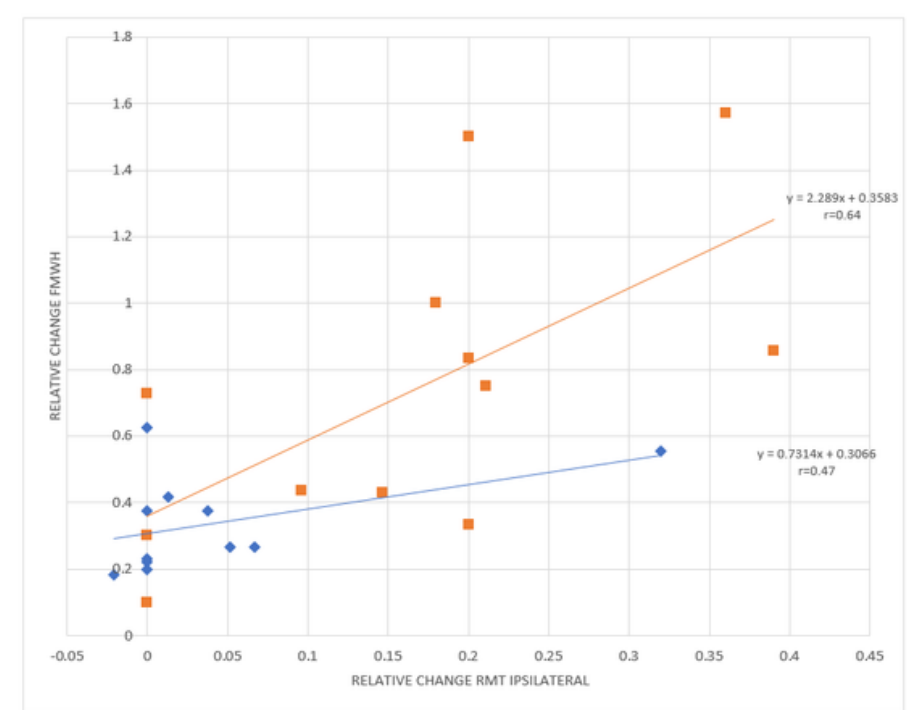

(A)

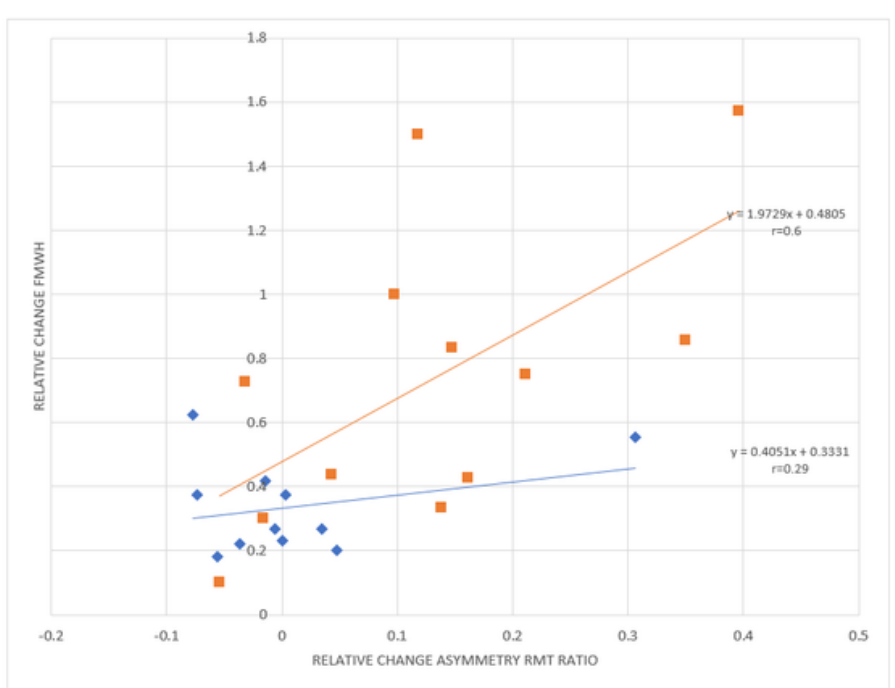

(B)

Figure 3

(A)Scatter-plot showing relationship between improvements in RMT in the ipsilesional-hemisphere and improvements in functional performance of the distal-component pre-to-post-therapy. Greater decreases in motor-threshold tend to show greater increases in FMW/H. Red Line (RG) and the blue line (CG) represents a linear-trend in improvement in distal motor-outcome $(\triangle \mathrm{FMW} / \mathrm{H})$ score as a function of change in the ipsilesionalhemisphere ( $\triangle \mathrm{RMTipsi)}$ in RG and CG pre-to-post-therapy in which RG shows a significantly strong correlation ( $r=0.64, F=7.24, p=0.022)$, (B) Scatter-plot showing relationship between change in RMT asymmetry-ratio (ipsilesional/contralesional) pre-to-post-therapy and functional performance of distal-component. Greater decreases in motor-threshold tend to show greater increases in FMW/H. Red line (RG) and the blue line (CG) represents a linear-trend in improvement in distal motor-outcome $(\Delta \mathrm{FMW} / \mathrm{H})$ score as a function of change in RMT-ratio $(\Delta \mathrm{RMTasymm}$ ratio) in $R G$ and $C G$ pre-to-post-therapy in which $R G$ shows a significantly strong correlation $(r=0.6, F=5.77, p=0.03)$ 